\title{
Protein Fluorescence Quenching by Small Molecules: Protein Penetration Versus Solvent Exposure
}

\author{
Dorothy B. Calhoun, ${ }^{1}$ Jane M. Vanderkooi, ${ }^{1}$ Gary R. Holtom, ${ }^{2}$ and S. Walter Englander ${ }^{1}$ \\ ${ }^{2}$ Department of Biochemistry and Biophysics and ${ }^{2}$ Department of Chemistry, University of Pennysylvania, \\ Philadelphia, Pennsylvania 19104
}

\begin{abstract}
Experiments were done to test the thesis that acrylamide and similar small molecules can penetrate into proteins on a nanosecond time scale. The approach taken was to measure the pattern of fluorescence quenching exhibited by quenching molecules differing in molecular character (size, polarity, charge) when these are directed against protein tryptophans that cover the whole range of tryptophan accessibility. If quenching involves protein penetration and internal quencher migration, one expects that larger quenchers and more polar quenchers should display lesser quenching. In fact, no significant dependence on quencher character was found. For proteins that display measurable quenching, the disparate quenchers studied display very similar quenching rate constants when directed against any particular protein tryptophan. For several proteins having tryptophans known to be buried, no quenching occurs. These results are not consistent with the view that the kinds of small molecules studied can quite generally penetrate into and diffuse about within proteins at near-diffusion-limited rates. Rather the results suggest that when quenching is observed, the pathway involves encounters with tryptophans that are partially exposed at the protein surface. Available crystallographic results support this conclusion.
\end{abstract}

Key words: proteins, protein dynamics, tryptophan exposure

\section{INTRODUCTION}

Proteins are by no means rigid, but engage in internal motions of many kinds. ${ }^{1-4}$ The amplitude and time scale of internal protein motions may be decipherable from the rates at which agents of varying molecular size can reach tryptophan side chains and quench their fluorescence or phosphorescence. Such encounters may reflect a deep penetration of the quencher into the protein matrix, a protein unfolding reaction that transiently exposes the tryptophan, or more simply some degree of tryptophan exposure to solvent in the native protein. The latter possibility is especially suggested by the fact that very few protein tryptophans are fully inaccessible to solvent. ${ }^{5,6}$

The surprising possibility that $\mathrm{O}_{2}$ might penetrate easily into proteins was first suggested by the studies of Lakowicz and Weber, ${ }^{7}$ which showed that $\mathrm{O}_{2}$ can quench the fluorescence of many protein tryptophans at nearly diffusion-limited rates. The Lakowicz-Weber thesis was challenged by others ${ }^{8}$ but was confirmed by Calhoun et al., ${ }^{9}$ who showed that $\mathrm{O}_{2}$ can indeed penetrate rapidly to the positions of some truly buried tryptophans. ${ }^{10}$

The possibility that more sizable agents like acrylamide may similarly penetrate into the protein matrix was raised by Eftink and Ghiron (reviewed in reference 11). In pursuing these issues we have adopted the hypothesis that the rate of true penetration reactions should be sensitive to molecular characteristics of the quencher such as size, polarity, and charge. This hypothesis seems self-evident in light of present knowledge concerning protein structure and is supported by a molecular dynamics simulation ${ }^{12}$ which displayed an extreme dependence of protein penetration rate on molecular size. Accordingly this paper describes studies of the fluorescence quenching efficiency of a number of dissimilar molecular agents when directed against a series of protein tryptophans having a wide range of accessibility.

\section{MATERIALS AND METHODS \\ Materials}

Proteins obtained from Sigma Chemical Co. in the highest available grade and used as provided included honey bee mellitin, $\mathrm{LADH}$, rabbit muscle aldolase, HSA, and RT1 from Aspergillus oryzae.

Parvalbumin was prepared from frozen cod fillets, locally obtained, by a modification of methods previously described. ${ }^{13,14}$ Frozen cod fillets $(125 \mathrm{~g})$ were homogenized with Tris buffer, $\mathrm{pH} 8.7$, in a blender, stirred for $1 \mathrm{hr}$, and centrifuged (10,000 rpm for 30 $\min )$. A $45-80 \%$ acetone cut $\left(0^{\circ} \mathrm{C}\right)$ was centrifuged; the pellet was resuspended in Tris buffer at $\mathrm{pH} 7.6$ in $1 \mathrm{mM} \mathrm{CaCl}$, heated rapidly $(\sim 5 \mathrm{~min})$ to $60^{\circ} \mathrm{C}$, and then immediately cooled and centrifuged. Subsequent steps were at $4^{\circ} \mathrm{C}$. The supernate was dialyzed against

Received June 11, 1986; accepted July 7, 1986.

Address reprint requests to $\mathrm{S}$. Walter Finglander, Depart ment of Biochemistry and Biophysics, University of Pennsylvania, Philadelphia, PA 19104.

Abbreviations used: AP, alkaline phosphatase; DEAE, diethyaminoethyl; EDTA, ethylene diamine tetraacetate; HSA, human serum albumin; LADH, horse liver alcohol dehydrogenase; MEK, methyl ethyl ketone; MVK, methyl vinyl ketone; NATA, $\mathrm{N}$-acetyl tryptophan amide; $\mathrm{PV}_{c}$ and $\mathrm{PV}_{n c}$, parvalbumin in absence and presence of calcium; RT1, ribonuclease $\mathrm{T} 1$. 
$1.6 \mathrm{mM}$ piperazine at $\mathrm{pH} 5.7$ and then chromatographed $(0.5 \mathrm{ml} / \mathrm{min})$ on a DEAE cellulose column $(2.5 \times 43 \mathrm{~cm})$ equilibrated with the same buffer. The column was washed until absorbance at $280 \mathrm{~nm}$ fell to 0.03 (ca. $24 \mathrm{hr}$ ) and then eluted with a $\mathrm{NaCl}$ gradient (800 $\mathrm{ml}, 0-0.1 \mathrm{M} \mathrm{NaCl}, 0.5 \mathrm{ml} / \mathrm{min}$ ), with protein detected by absorbance at $280 \mathrm{~nm}$. Peaks of the two parvalbumin species were separated, and the tryptophan-containing species was dialyzed against water and lyophilized. Fluorescence quenching experiments were done with parvalbumin either containing its normal bound calcium $\left(\mathrm{PV}_{\mathfrak{c}}\right)$ or in the presence of $2 \mathrm{mM}$ EDTA to remove thte calcium ( $\left.P V_{n c}\right)$.

Among the quenchers used, acrylamide and MVK were from Sigma and bisacrylamide from Eastman Kodak. HPLC grade acetone, acrylic acid, and other reagent grade chemicals were from Fisher Scientific. The solvent used was $0.01 \mathrm{M}$ sodium phosphate at $\mathrm{pH}$ 7.0 in $0.1 \mathrm{M} \mathrm{NaCl}$ except for studies with mellitin where a low salt condition was used $(0.01 \mathrm{M}$ Tris at $\mathrm{pH} \mathrm{8.0)}$ to maintain the unstructured state.

\section{Quenching Measurements}

Steady-state fluorescence spectra and intensities in most of the quenching experiments were measured with a SLM-8000 fluorimeter interfaced to a HewlettPackard 9825 computer. Oxygen quenching experiments were done in a Perkin-Elmer 650 fluorimeter adapted to accept a high-pressure stainless-steel cell chamber previously described. ${ }^{9}$ In the quenching experiments an excitation wavelength of $300 \mathrm{~nm}$ was used to avoid contributions from tyrosine fluorescence. Emission was measured at either 325 or 350 $\mathrm{nm}$ depending on the protein in order to avoid the Raman peak (at $334 \mathrm{~nm}$ ). Protein concentration was usually adjusted to provide optical density -0.1 at $300 \mathrm{~nm}$.

Since some of the qenching agents, used at high concentration, have significant absorbance at the excitation and emission wavelengths, inner filter corrections to the fluorescence intensity data were necessary, as previously described. ${ }^{15}$ Minor fluorescence background due to some of the quenchers was measured in parallel experiments set up to replicate the protein experimental conditions, but without protein present. These values were subtracted from the protein data before applying inner filter corrections.

\section{Data Analysis}

Values for quenching rate constant $\mathrm{k}_{\mathrm{q}}$, listed in Table I) were obtained from steady-state fluorescence intensity measurements, as is true of almost all the work leading to the penetration hypothesis tested here. Quenching rate constants were computed from the Stern-Volmer equation (Eq. 1).

$$
F_{0} / F=1+k_{q} t_{0}[Q]
$$

Here $\mathrm{F}_{0}$ represents steady-state fluorescence intensity in the absence of quencher and $F$ in the presence of quencher at concentration [Q]. The parameter $F_{0} / F-1$ often increases linearly with [Q], in which case the rate constant $\mathrm{k}_{\mathrm{q}}$ can be obtained from the (initial) slope of a linear Stern-Volmer plot (Figs. 13).

It can be noted that measurements like these do not distinguish dynamic collisional quenching from quenching due to ground state complexation. This does not reflect on the thesis of this paper. If the results found here are to be explained in terms of ground state complexation, this by definition agrees with the conclusion reached, that the quenching observed does not represent rapid dynamic penetration. Experiments dealing directly with this issue will be presented elsewhere.

\section{RESULTS}

Studies were done to measure the ability of various small molecule agents to quench the singlet excited state of a number of protein tryptophans. Typical quenching data are exhibited in Figures 1-3, and results are summarized in Table I.

TABLE I. Fluorescence Quenching Constants $\left(M^{-1} \mathbf{n s}^{-1}\right)$ *

\begin{tabular}{lccccccc}
\hline Quencher & NATA & Mellitin & PV $_{\text {nc }}$ & LADH & PV $_{\mathbf{c}}$ & RT1 & Aldolase \\
\hline $\mathrm{O}_{2}$ & 15 & & 5 & 3 & 2 & 2 & 3 \\
Nitrite & 13 & 40 & 8 & 2 & 4 & 4 & 2 \\
MVK & 13 & 4 & 4 & 3 & 2 & 1.5 & $\sim 0$ \\
Nitrate & 10 & 23 & 0.5 & 0.4 & 0.2 & -0.01 & -0.01 \\
Acrylate & 3 & & 1 & 0.7 & 0.3 & 0.06 & -0 \\
Acrylamide & 9 & 4 & 2 & 0.8 & 0.1 & 0.25 & 0.06 \\
Acetone & 4 & 2 & 0.7 & 0.6 & 0.1 & -0.02 & -0.04 \\
MEK & 5 & 3 & 0.9 & 0.6 & 0.2 & 0.07 & -0.04 \\
\hline
\end{tabular}

*Quenching rate constants, listed in units of $\mathrm{M}^{-1} \mathrm{~ns}^{-1}$, were measured by decrease in fluorescence intensity as a function of quencher concentration. Fluorescence lifetimes used to calculate the quenching

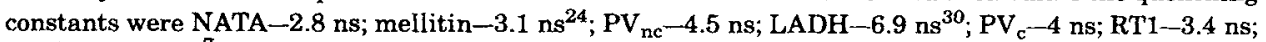
aldolase--2.3 ns. ${ }^{7}$ The proteins generally display a multicomponent decay; the lifetime used in each case represents a qualitative average value that reasonably represents, for present purposes, the major fraction of the emitted intensity. For LADH, which has two tryptophans per subunit, Stern-Volmer plots plateaued at high quenching and $\mathrm{k}_{\mathrm{q}}$ was computed for the quenchable fraction. In experiments with aldolase, protein concentration was $\sim 1 \mathrm{mg} / \mathrm{ml}$. 


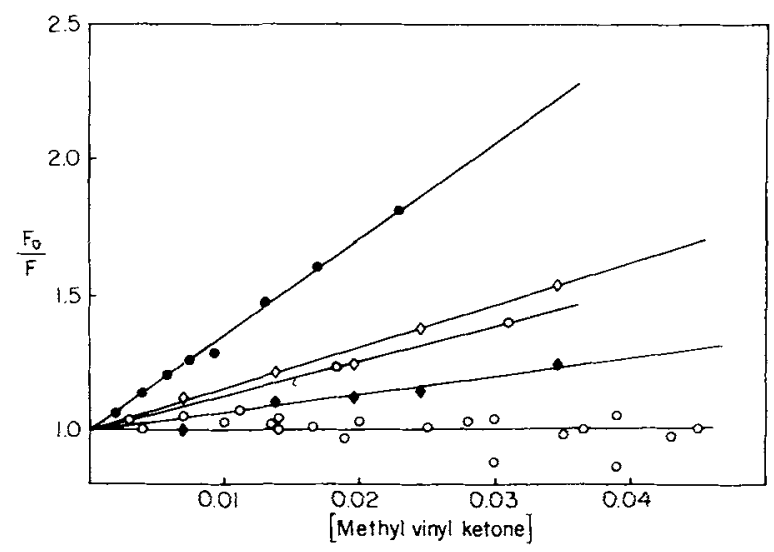

Fig. 1. Stern-Volmer plot of fluorescence quenching by MVK, measured by decrease in steady-state emission intensity. The fluors studied are represented by the following symbols: - NATA; $O$, mellitin; $\diamond, P V_{n c} ; \bullet, P V_{c} ; \square, R T 1 ; O$, aldolase.

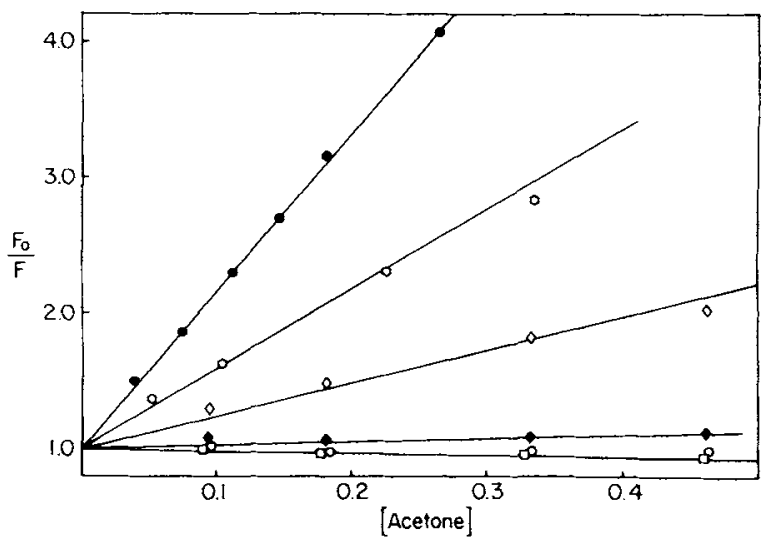

Fig. 2. Stern-Volmer plot of fluorescence quenching by acetone, measured by decrease in steady-state emission intensity. Symbols are as in Figure 1.

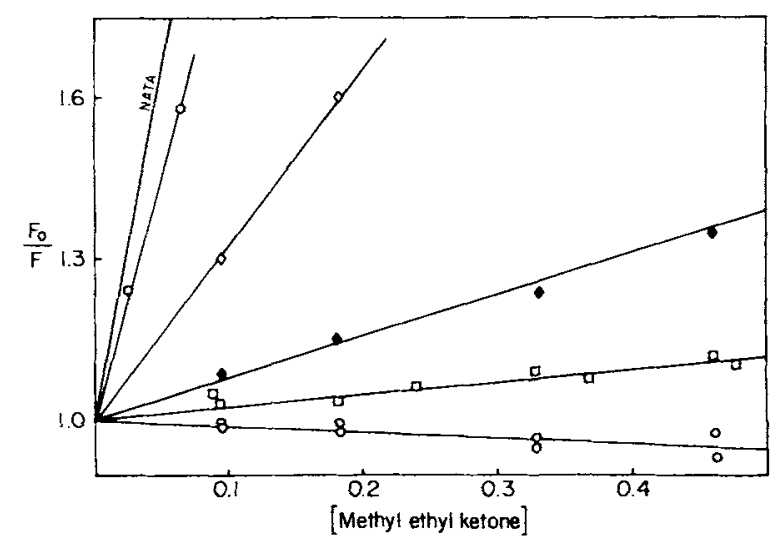

Fig. 3. Stern-Volmer plot of fluorescence quenching by MEK, measured by decrease in steady-state emission intensity. Symbols are as in Figure 1.

\section{Fluors and Quenchers Studied}

Among the fluors studied, we used the fully exposed indole of $\mathrm{N}$-acetyl tryptophan amide (NATA) to identify a number of high efficiency quenchers, with rate constants at or near the diffusional limit. Proteins covering the whole range of tryptophan accessibility were studied, with emphasis on those that appear to have the most protected indoles in order to maximize the chance of observing true protein penetration reactions. These include the proteins considered by Eftink \& Ghiron to display penetration-dependent fluorescence quenching, except for human serum albumin, which is well known to bind agents like the quenchers studied here.

Several classes of quenching agents were studied. The small apolar molecule, $\mathrm{O}_{2}$, quenches the fluorescence of fully exposed tryptophan at a diffusion limited rate and has been found ${ }^{7,9}$, to quench a variety of protein tryptophans at nearly the same rate, indicating an ability to penetrate rapidly into proteins. This holds also for the proteins studied here (Table I). The results with $\mathrm{O}_{2}$ can be taken as an indication of the quenching behavior to be expected for agents that can penetrate easily into proteins. That is, incoming $\mathrm{O}_{2}$ is insensitive to molecular barriers that effectively block the larger quenchers (Table I).

The resonance energy transfer agents, MVK and $\mathrm{NO}_{2}{ }^{-}$anion, have significant optical absorbance overlapping the indole fluorescence emission band, leading to a small Forster transfer parameter, $R_{0}$, of about $10 \AA .{ }^{15} \mathrm{MVK}$ and $\mathrm{NO}_{2}{ }^{-}$can quench tryptophan fluorescence from a distance of several angstroms, and this contributes to their quenching capability. These agents are chemical analogs of the contact quenchers used (see below), but they maintain much higher $k_{\mathrm{q}}$ values against the less accessible tryptophans (Table I). This is so in spite of their small Forster $R_{0}$ parameters $(10 \AA)$, providing evidence that all the tryptophans studied extend quite close to the protein surface.

Given the results with $\mathrm{O}_{2}$ and the transfer agents as background, we are here mainly interested in the possible ability of molecules like acrylamide to penetrate into and diffuse rapidly within proteins. ${ }^{11}$ To study this we used a series of small molecule contact quenchers with varying size, polarity, and charge, which can be expected to affect their penetrational capability. These include acrylamide itself; the ketones methyl methyl ketone (acetone) and MEK; acrylate, which is a charged analog of acrylamide; and the smaller nitrate anion. These quenching agents have negligible spectral overlap with tryptophan emission and require essentially a van der Waals contact with the target tryptophan in order to quench the tryptophan excited state. On contact, quenching processes in addition to dipolar Forster transfer may act. ${ }^{16,17}$

\section{Exposed Tryptophan: NATA and Mellitin}

Against NATA, a freely exposed indole model, the rapidly diffusing $\mathrm{O}_{2}$ molecule displays a diffusionlimited quenching rate constant of $15 \mathrm{M}^{-1} \mathrm{~ns}^{-1}$ (Table I). A number of larger agents show similar $\mathrm{k}_{\mathrm{q}}$ values (Table I), close to the diffusional limit. 
Mellitin (Table I), a 26-residue polypeptide with one tryptophan, was studied at low concentration $(\sim 1$ $\mathrm{mg} / \mathrm{ml}$ ) in low salt conditions to ensure the monomeric, random coil state ${ }^{18}$ and major exposure of the tryptophan residue. The nonionic quenchers show similar quenching rate constants against mellitin, about one-half that found for the freely exposed NATA fluor (Table I). The anionic quenchers, nitrite and nitrate (and iodide, not shown), display exaggerated quenching ability, exceeding by severalfold the diffusion-limited rate seen for NATA, presumably due to charge attraction since the single tryptophan in mellitin is adjacent to a sequence of positively charged residues (see also reference 31 ).

\section{Moderately Accessible Tryptophans-PV and LADH}

The proteins studied are listed in Table I from left to right in order of decreasing sensitivity to quenching. Mellitin and PV have a single tryptophan residue. Parvalbumin was studied with bound calcium ion $\left(\mathrm{PV}_{\mathrm{c}}\right)$ and in the presence of EDTA $\left(\mathrm{PV}_{\mathrm{nc}}\right)$ which chelates the structural calcium of PV, making its tryptophan residue more accessible. LADH has one relatively exposed tryptophan residue per subunit, $, 8,19$ which is responsible for the quenching observed, and one well-buried tryptophan not accessible to these quenchers (see below). This produces concave downward Stern-Volmer plots for LADH quenching. In this case $k_{\mathrm{q}}$ values for the more accessible tryptophan can be computed from Lehrer inverse plots. ${ }^{15,20}$

The contact quenchers- $\mathrm{NO}_{3}{ }^{-}$, acrylate, acrylamide, acetone, and MEK-all display very similar ability to reach any given tryptophan (Table I) in spite of the fact that the quenchers differ considerably in size and charge. A narrow range is seen for $P V_{n c}$, which is a step down in accessibility from mellitin. Results for the more accessible of the two LADH tryptophans are tightly grouped with $\mathrm{k}_{\mathrm{q}}$ for the different agents all between 0.4 and $0.8 \mathrm{M}^{-1} \mathrm{~ns}^{-1}$. Published results show that bisacrylamide, effectively a double-sized acrylamide molecule, has a nearly identical $k_{\mathrm{q}}$ of 1 $M^{-1} \mathrm{~ns}^{-1} .{ }^{15}$ In the presence of calcium $\left(\mathrm{PV}_{\mathrm{c}}\right)$, the single tryptophan in PV becomes distinctly more protected, but again, all the contact quenchers reach this tryptophan with $\mathrm{k}_{\mathrm{q}}$ in a tight range, about 0.2 $\mathrm{M}^{-1} \mathrm{~ns}^{-1}$, approaching the measurable limit.

In summary, taking NATA as a fully exposed standard, the apparent protection factor against the contact quenchers for the proteins so far discussed is roughly 2 for mellitin, 4 for $\mathrm{PV}_{\mathrm{nc}}, 10$ for $\mathrm{LADH}$, and 40 for $P V_{c}$. In each protein, the $k_{q}$ values found for the very different contact quenchers are impressively similar in spite of their different sizes and even though some (nitrate, acrylate) are ionic. This is not the pattern expected for a penetrational pathway. The fact that a given tryptophan exhibits similar accessibility to quite different quenchers suggests that the tryptophan is still partially exposed at the protein surface.

To search for significant protein penetration, it therefore appears necessary to study tryptophans with still lower accessibility, i.e., with $\mathrm{k}_{\mathrm{q}}$ less than $2 \times 10^{8}$ $\mathbf{M}^{-1} \mathbf{s}^{-1}$. Only a few known proteins produce $k_{q}$ values that low.

\section{Nonexposed Tryptophans}

The single tryptophan in azurin is known from $\mathrm{x}$ ray diffraction studies ${ }^{21}$ to be fully buried. Eftink and Ghiron $^{22}$ have shown that fluorescence of the azurin tryptophan is not measureably quenched by acrylamide. We confirm this result.

Other proteins known to have a well-buried tryptophan have also some more accessible tryptophans, which renders sensitive fluorescence quenching measurements of the buried tryptophan difficult. However, a related approach, the time-resolved measurement of room temperature phosphorescence quenching, ${ }^{8}$ which focuses specifically on well-protected tryptophans, then becomes most useful since only the buried tryptophan contributes to the measured phosphorescence. We have studied ${ }^{9,15} \mathrm{LADH}$ and AP, which show remarkably long lifetimes for room temperature phosphorescence $(0.35 \mathrm{~s}$ and $1.5 \mathrm{~s}$, respectively). A variety of contact quenchers including some used here can quench the phosphorescence of the buried tryptophan in LADH and in AP, but this occurs with rate constants that are many orders of magnitude below those measured here (ca. $10^{-4}$ and $10^{-8} \mathrm{M}^{-1} \mathrm{~ns}^{-1}$, respectively; measurable ${ }^{15}$ on the much slower phosphorescence time scale).

In short, studies of the truly buried tryptophans of azurin, $\mathrm{LADH}$, and AP unambiguously rule out fast protein penetration by the kinds of small molecules studied here. It can be noted that $\mathrm{O}_{2}$ reaches these tryptophans with a "normal" rate constant, $\sim 10^{9}$ $\mathbf{M}^{-1} \mathbf{s}^{-1.9}$ Thus the blockage of the other contact quenchers does not appear to reflect some atypical structural barrier, but merely the fact of full tryptophan burial within the protein.

\section{Tryptophans With Questionable Exposure: RT1 and} Aldolase

The known repertoire of proteins with quenching rate constants smaller than $0.2 \mathrm{M}^{-1} \mathrm{~ns}^{-1}$ but still measurably greater than zero include only RT1 and aldolase. Data on the quenching of these two proteins by acrylamide supply a major part of the evidence previously interpreted as demonstrating the penetrability of acrylamide into proteins. ${ }^{11}$ Results for the quenchers we tested are in Table I.

$\mathrm{O}_{2}$ quenches RT1 and aldolase fluorescence about as well as other proteins. Quenching by the resonance energy transfer agent, $\mathrm{NO}_{2}{ }^{-}$, suggests that the RT1 and aldolase tryptophans are still within a few angstroms of the surface, though the $k_{q}$ for MVK suggests 
some decreased accessibility, especially in aldolase. Acrylamide quenches $\mathrm{RT} 1$ with an apparent $\mathrm{k}_{\mathrm{q}}$ value of $0.25 \mathrm{M}^{-1} \mathrm{~ns}^{-1}$ and aldolase with $\mathrm{a} \mathrm{k}_{\mathrm{q}}$ of 0.1 $\mathrm{M}^{-1} \mathrm{~ns}^{-1}$ (measured from the initial part of an upward curving Stern-Volmer plot, Fig. 4). These results duplicate the earlier findings of Eftink and Ghiron. ${ }^{23}$ The other contact qenchers studied have $k_{q}$ s for these proteins differing only marginally if at all from zero. Clearly the contact quenchers do not penetrate into these proteins at a rate near the free-solvent diffusional limit.

Various mechanisms for the enhanced activity of acrylamide against $\mathrm{RT} 1$ and aldolase (in comparison with the other contact quenchers) can be considered. If we view the immediate tryptophan environment in the sense of solvent, the significant effect of the local surround on quenching efficiency, described by Eftink and Ghiron, ${ }^{24}$ may conceivably exercise a selective effect on the varying quenchers. Another interesting possibility is suggested by the ideas of Berg ${ }^{25}$ and Berg and von Hippel ${ }^{26}$ on protein surface diffusion. Perhaps acrylamide can bind nonspecifically to the protein surface and diffuse over it to produce a more efficient encounter with the occluded tryptophan side chain. In the case of aldolase, a possible contributing factor rests on its tendency to dissociate into monomers. Rabbit muscle aldolase is a tetramer of nearly identical subunits, each having three tryptophan res-

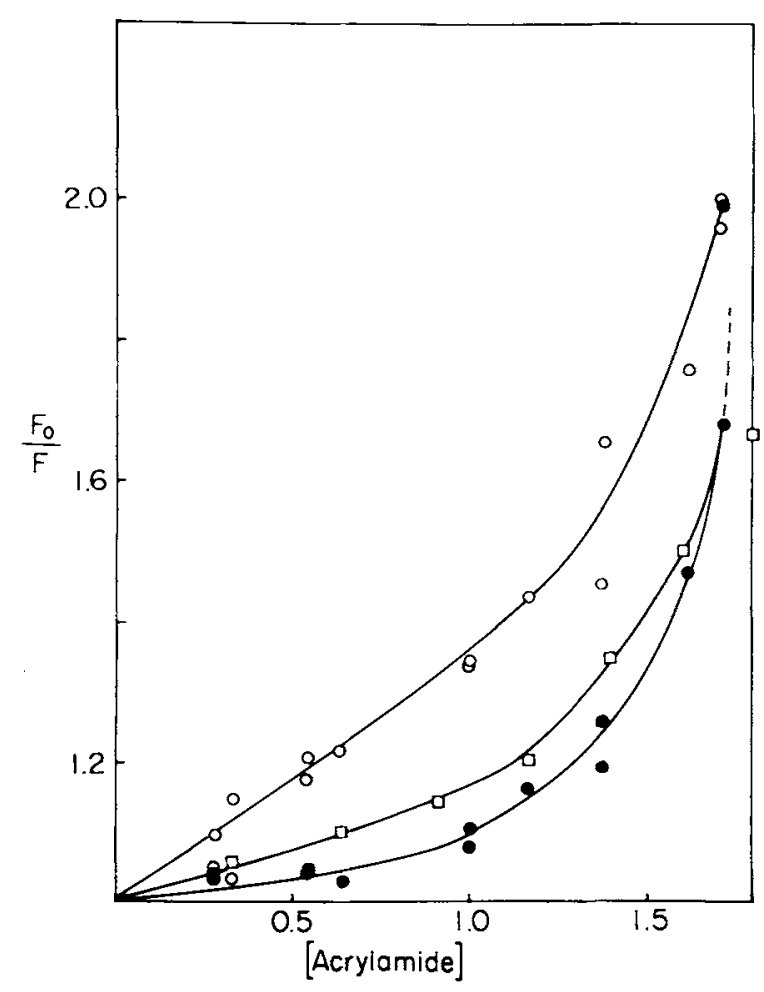

Fig. 4. Stern-Volmer plot showing fluorescence quenching of aldolase by acrylamide, measured by decrease in steady-state emission intensity. Concentration of aldolase was $0.08 \mathrm{mg} / \mathrm{ml}$ (O), $0.3 \mathrm{mg} / \mathrm{ml}(\square)$, and $1.4 \mathrm{mg} / \mathrm{ml}(0)$. idues. $^{27}$ Stellwagen and Schachman ${ }^{28}$ have shown that aldolase dissociates at slightly acid $\mathrm{pH}$, being half-dissociated at $\mathrm{pH}$ 5.5. Figure 4 indicates that aldolase becomes more susceptible to quenching at lower protein concentration, consistent with a contribution from protein dissociation. A structural effect due to weak acrylamide binding is suggested by the remarkably sharp upsweep in quenching at high acrylamide concentration seen in Figure 4. Perhaps acrylamide promotes aldolase dissociation and a consequent exposure of its tryptophans. Eftink and Ghiron $^{22}$ noted the ability of acrylamide to bind to aldolase at the high concentrations used in these experiments and found some denaturation-like changes above $0.5 \mathrm{M}$ acrylamide. The aberrant interaction of acrylamide with RT1 and aldolase is interesting and deserves further investigation.

For the questions addressed in the present study, the near-zero quenching ability found for the other contact quenchers studied seems most indicative. Whatever other undescribed events may be occurring, this result is clearly inconsistent with the thesis that such molecules can penetrate rapidly into proteins.

\section{DISCUSSION}

A great deal of discussion has now been devoted to the apparent plasticity of proteins. One possible way to study these issues is by observation of the movement of small molecules into and through proteins. This approach is in some ways analogous to the observation of molecular diffusion in lipid bilayers that earlier helped to define the liquid-like character of biomembranes. As a first step one wants to discover the kinds of molecules that can move into proteins and to measure their rates of intrusion and internal migration. It is now clear that molecular oxygen can penetrate, or at least insert, into proteins and quench truly buried tryptophans at a rate only a little lower than the free solvent diffusional limit. ${ }^{7,9,10}$ Can other molecules do the same?

\section{Prior Results}

The possibility that larger molecules like acrylamide might move rapidly into and through proteins has been reviewed by Eftink and Ghiron. ${ }^{11}$ The major evidence adduced in favor of this conclusion involves the temperature and viscosity dependence of the quenching of a number of proteins by acrylamide. Measured temperature dependences indicate activation energy for the quenching process ranging from 2 to $11 \mathrm{kcal} \mathrm{M}^{-1}$ for various proteins, compared to about $4 \mathrm{kcal}$ for the fully exposed NATA model. Interpretation of this small range of values in terms of molecular mechanism is problematic and is complicated by the possible intervention of various phenomena such as protein-quencher binding, especially at the high quencher concentrations necessary for these experiments $(\sim 0.1-1 \mathrm{M})$. The observation of a correIation between decreasing $k_{q}$ values among various 
proteins and decreasing dependence on solvent viscosity has similarly been taken to suggest that the ratelimiting event in the quenching process occurs within the protein. However, this kind of observation is also clouded by the possible role of alternative processes, including protein binding (to be discussed elsewhere).

\section{Method of Investigation}

In this work we utilized an approach that can provide direct information on the penetration question. To implement this approach we identified a number of small molecule agents that can quench the fluorescence of free tryptophan with nearly diffusion-limited rates. The different agents differ significantly in molecular size and polarity. The experiments were intended to discern the pattern of quenching exhibited by the range of quenchers when they are tested against a number of protein tryptophans that differ greatly in accessibility.

We will refer to the behaviour shown by $\mathrm{O}_{2}$, which experiences very little resistance in reaching masked tryptophans, as the pattern of the first kind. $\mathrm{O}_{2}$ encounters the tryptophans of the proteins in Table I with rate constants near the diffusional limit and within the narrow range $5 \mathrm{M}^{-1} \mathrm{~ns}^{-1}$ to $2 \mathrm{M}^{-1} \mathrm{~ns}^{-1}$. The same result has been found for a large number of other proteins. ${ }^{7}$ For the most effectively shielded tryptophans, the truly buried tryptophans of $\mathrm{LADH}$ and alkaline phosphatase (which display room temperature phosphorescence lifetimes of 0.4 and $1.5 \mathrm{~s}$, respectively) the quenching rate constant for $\mathrm{O}_{2}$ is reduced a little more, to $1 \mathrm{M}^{-1} \mathrm{~ns}^{-1} .^{9}$

Against the same sequence of target proteins, the encounter rate for the other contact quenchers studied here falls off more sharply, reaching negligibly small values for the most protected tryptophans when measured by fluorescence quenching. The encounter rate for some of these same agents with the truly buried tryptophans in LADH and alkaline phosphatase can be seen to fall by a factor of $10^{4}-10^{8}$ or more when measured in phosphorescence experiments. ${ }^{15}$ Clearly these quenchers do not achieve penetration behavior of the first kind. Unlike the situation with $\mathrm{O}_{2}$, molecular barriers effectively inhibit their approach to target tryptophans. This is hardly surprising. A computer simulation of protein penetration behavior due to Richards ${ }^{12}$ showed an extreme sensitivity to the size of the penetrating molecule, and the barrier against partitioning polar and, most especially, ionic groups into the protein interior has been obvious since protein crystallographic models first became available.

If these quenching agents can in fact penetrate into proteins at all, they must therefore be expected to display a pattern of the second kind, in which larger and more polar molecules are more effectively blocked. Thus, if protein penetration provides the pathway for quenching, one expects that methyl ethyl ketone should be less effective than acetone (methyl methyl ketone), the more polar acrylamide might be still less effective, and the ionic agents, acrylic acid and nitrate, should be excluded from the hydrophobic protein interior.

\section{Results Obtained}

The results obtained exhibit a quenching pattern very different from the one expected for protein penetration. Impressively similar quenching rate constants were observed for the various quenchers when tested against any given tryptophan; major differences in quencher character seemed irrelevant. These resuits make it clear that the quenching pathway is not rate-limited by a through-protein penetration. The pattern found is what one expects if the tryptophan being quenched is partially exposed to the solvent. The different quenchers can then all encounter the tryptophan with approximately the same decreased rate. This pattern held for proteins exhibiting quenching rate constants down to about $0.2 \mathrm{M}^{-1} \mathrm{~ns}^{-1}$. Apparently quenching constants down to this level still indicate partially exposed tryptophans. Available x-ray diffraction results for LADH agree. ${ }^{21}$ Thus any contribution due to actual protein penetration must have a rate constant lower than $0.2 \mathrm{M}^{-1} \mathrm{~ns}^{-1}$.

In other experiments, several proteins having tryptophans known to be fully buried have been tested by us and others. ${ }^{15,24,29}$ For these proteins, fluorescence quenching rate constants are effectively zero. This is so in spite of the fact that these tryptophans exhibit "normal" quenchability by $\mathrm{O}_{2}$ and project close to the protein surface, as indicated by their sensitivity to quenching by resonance energy transfer agents with small characteristic transfer distance. Thus the results with the contact quenchers document the absence of fast quencher penetration, or even significant quencher insertion, in these cases.

Only two proteins are known (to us) with rate constants less than $0.2 \mathrm{M}^{-1} \mathrm{~ns}^{-1}$ but still measurably greater than zero. These are RT1 and aldolase. The fluorescence of RT1 displays minimal, barely measurable quenching by the agents tested, with the exception of acrylamide. Again here, results with the energy transfer quenchers indicate that the RT1 tryptophan is close to the protein surface. Emerging crystallographic results similary indicate (W. Saenger, personal communication) that the lone tryptophan indole of RT1 is not totally buried but an edge is just marginally accessible. Thus the near-zero RT1 quenching rates observed for most of the contact quenchers stand against the view that such agents can move deeply into this protein in nanoseconds. Even the low level of quenching observed seems able to be explained by residual exposure of the tryptophan at the protein surface rather than by penetration into the protein interior.

Finally, in the case of aldolase the contact quenchers fail to achieve measurable quenching, except for acrylamide. The results in Figure 4 suggest that at 
the high concentrations of acrylamide used (0.1-1.5 M), some induced protein subunit dissociation occurs with consequent exposure of the tryptophan. In any case the aldolase quenching results with the other contact quenchers show that rapid penetration into this protein does not occur.

\section{Conclusion}

In summary, for the kinds of quenching agents studied here, the present results are against a significant role for rapid protein penetration in the quenching mechanism. The results suggest that protein quenching rate constants larger than $0.2 \mathrm{M}^{-1} \mathrm{~ns}^{-1}$ identify partially exposed tryptophans and reflect quencher-tryptophan encounters at the protein surface. This accounts for almost the whole range of rate constants available to fluroescence quenching measurement. Some tryptophans known to be fully buried (in azurin, $\mathrm{LADH}$, and $\mathrm{AP}$ ) have a quenching rate constant of zero and thus are clearly not reached and quenched by these agents on the fluorescence time scale. For the few proteins that fall in the narrow range between these groups (ribonuclease $\mathrm{T} 1$ and aldolase), most quenchers are ineffective, again ruling out rapid penetration.

\section{ACKNOWLEDGMENTS}

This work was supported by NIH research grants GM 21487 and AM 11295

\section{REFERENCES}

1. Gurd, F.R.N., Rothgeb, M. Motions in proteins. Adv. Protein Chem. 33:73-165, 1979

2. Karplus, M., McCammon, J.A. The internal dynamics of globular proteins. CRC Crit. Rev. Biochem. 9:293-349, 1981

3. Sarma, R.H. "Biomolecular Stereodynamics." New York: Adenine Press, 1981

4. Englander, S.W., Kallenbach, N.R. Hydrogen exchange and structural dynamics of proteins and nucleic acids. $Q$. Rev. Biophys. 16:521-655, 1984.

5. Chothia, $C$. The nature of the accessible and buried sur faces in proteins. J. Mol. Biol. 105:1-14, 1976.

6. Guy, H.R. Amino-acid side-chain partition energies and distribution of residues in soluble proteins. Biophys. J. 47:61-70, 1985.

7. Lakowicz, J.R., Weber, G. Quenching of protein fluorescence by oxygen. Detection of structural fluctuations in proteins on the nanosecond time scale. Biochemistry 12:4171-4179, 1973 .

8. Saviotti, M.L., Galley, W.C. Room temperature phosphorescence and the dynamic aspects of protein structure. Proc. Nat. Acad. Sci. USA 71:4154-4158, 1974.

9. Calhoun, D.B., Vanderkooi, J.M., Woodrow, G.V. III, Englander, S.W. Penetration of dioxygen into proteins studied by quenching of phosphorescence and fluorescence. Biochemistry 22:1526-1532, 1983.
10. Gratton, E., Jameson, D.M., Weber, G. A model of dynamic quenching of fluorescence in globular proteins. Biophys $\mathrm{J}$. 45:789-794, 1984.

11. Eftink, M.K., Giron, C.A. Review of fluorescence quenching studies with proteins. Anal. Biochem. 114:199-227, 1981.

12. Richards, F.M. Packing defects, cavities, volume fluctuations, and access to the interior of proteins. CarIsberg Res. Commun. 44:47-63, 1979.

13. Haiech, J., DeRancourt, J., Pechere, J.F., DeMaille, J.G. A new large-scale purification procedure for muscular parvalbumins. Biochimie 61:583-587, 1979.

14. Harrocks, W.D. Jr., Collier, W.E. Lanthanide ion luminescence probes. Measurement of distance between protein fluorophores and bound metal ions: quantitation of energy transfer between tryptophan and terbium(III) or europium(II) in the calcium-binding protein parvalbumin. $J$. Am. Chem. Soc. 103:2856-2862, 1981.

15. Calhoun, D.B., Vanderkooi, J.M., Englander, S.W. Penetration of small molecules into proteins studied by quenching of phosphorescence and fluorescence. Biochemistry 22:1533$1539,1983$.

16. Turro, N.J. "Modern Molecular Photochemistry." Menlo Park, CA: Benjamin-Cummings. 1978:296-361.

17. Lakowicz, J.R. "Principles of Fluorescence Spectroscopy." New York: Plenum Press, 1983

18. Bello, J., Bello, H.R., Granados, E. Conformation and aggregation of melittin: dependence on $\mathrm{pH}$ and concentration. Biochemistry 21:461-465, 1982.

19. Branden, C.I., Jornvall, H., Ekiund, H., Furugren, B. Alcohol dehydrogenases. In: "The Enzymes." 3rd Ed., Vol. 11 a. New York and London: Academic Press. 1975:103-190.

20. Lehrer, S.S. Solute perturbation of protein fluorescence. The quenching of the tryptophyl fluorescence of model compounds and of lysozyme by iodide ion. Biochemistry 10:3254-3263, 1971 .

21. Adman, E.T., Stenkamp, R.E., Sieker, L.C., Jensen, L.H. A crystallographic model for azurin at $3 \mathrm{~A}$ resolution. J. Mol. Biol. 123:35-47, 1978.

22. Eftink, M.K., Ghiron, C.A. Exposure of tryptophanyl residues in proteins. Quantitative determination by fluorescence quenching studies. Biochemistry 15:672-680, 1976.

23. Eftink, M.K., Ghiron, C.A. Exposure of tryptophanyl residues and protein dynamics. Biochemistry 16:5546-5551, 1977.

24. Eftink, M.K., Ghiron, C.A. Indole fluorescence quenching studies on protein and model systems: use of the inefficient quencher succinimide. Biochemistry 16:5546-5551, 1984.

25. Berg, $O$. Orientation constraints in diffusion-limited macromolecular association. Biophys. J. 47:1-14, 1985.

26. Berg, O., von Hippel, P.H. Diffusion-controlled macromolecular interactions. Annu. Rev. Biophys. Biophys. Chem. 14:131-160, 1985 .

27. Lai, C.Y., Nakai, N., Chang, D. Amino acid sequence of rabbit muscle aldolase and the structure of the active center. Science 183:1204-1206, 1974.

28. Stellwagen, E., Schachman, H.K. The dissociation and reconstitution of aldolase. Biochemistry 1:1056-1069, 1962.

29. Fftink, M.K. Quenching-resolved emission anisotropy studies with single and multitryptophan-containing proteins. Biophys. J. 43:323-334, 1983.

30. Eftink, M.K., Jameson, D.M. Acrylamide and oxygen fluo rescence quenching studies with liver alcohol dehydrogen ase using steady state and phase fluorometry. Biochemistry 21:4443-4449, 1982.

31. Wensel, T.G., Meares, C.F. Electrostatic properties of myoglobin probed by diffusion-enhanced energy transfer. Biochemistry 22:6247-6254, 1983. 